\title{
COMPARISON OF CLINICAL FAILURES OF LINGUAL RETAINERS BONDED WITH PRIMERLESS FLOWABLE ADHESIVES VERSUS CONVENTIONAL BONDING ADHESIVES
}

\author{
Faheem Nake Akhtar, Abdullah Jan, Sana Tariq, Mehwish Khan, Munazza Saeed \\ Armed Forces Institute of Dentistry/National University of Medical Sciences (NUMS) Rawalpindi Pakistan
}

\begin{abstract}
Objective: To compare the frequency of clinical failure of lingual retainers bonded with primerless flowable adhesives and conventional bonding adhesives.

Study Design: Quasi experimental study.

Place and Duration of Study: Department of Orthodontics, Armed Forces Institute of Dentistry Rawalpindi, from Jul 2017 to Jun 2018.

Methodology: As per selection criteria, 76 patients were selected by non-probability consecutive sampling technique. Patients were divided into two groups: the conventional bonding adhesive and the primerless flowable adhesive group with 38 patients in each group. Retainers were bonded using standardized procedure with conventional bonding adhesive in one group and primerless flowable adhesive for bonding in the other group. After bonding of retainers, patients were recalled after 3 months and any bonding failures were recorded.

Results: Failure rate with conventional bonding adhesive was $10.5 \%$ while on the other hand, failure with primerless flowable adhesive was $31.6 \%$ with a $p$-value of 0.047 which showed a statistically significant difference.

Conclusion: There was a statistically significant difference of clinical failure rate of lingual retainers bonded with conventional bonding adhesive versus primerless flowable adhesive with greater failure rate of the primerless flowable adhesive.
\end{abstract}

Keywords: Conventional bonding adhesives, Lingual retainer failure, Primer, Primerless flowable adhesives.

This is an Open Access article distributed under the terms of the Creative Commons Attribution License (http://creativecommons.org/licenses/by/4.0), which permits unrestricted use, distribution, and reproduction in any medium, provided the original work is properly cited.

\section{INTRODUCTION}

Sustaining the teeth in their final position after orthodontic therapyis probably one of the greatest challenges for the orthodontist since there is a predisposition for relapse resulting in loss of achieved treatment objectives ${ }^{1}$.

Retainers are therefore considered an indispensable part of treatment and are necessary to resist this tendency for relapse. For this purpose, lingual retainers are used, which include multistr and wires that with the help of composite adhesives areadheredonto the lingual areas of the lower incisors, resulting in the provision of an effective structure for retention ${ }^{2}$.

Retainers are attached to the tooth surface using composite resin adhesives. The adhesives that are currently being used for bonding to enamel surfaces, have progressed a long way since their inception in the 1950s by Buonocore ${ }^{3}$. The conventional composites that are frequently used require three steps in placement: an initial step that involves etching of the enamel surface, followed by application of a primer layer, and the final step being placing the adhesive along with light curing. These conventional composites require an

Correspondence: Dr Faheem Nake Akhtar, Orthodontics Department, Armed Forces Institute of Dentistry, Rawalpindi Pakistan

Received: 02 Dec 2019; revised received: 27 Dec 2019; accepted: 10 Jan 2020 additional step of primer application which causes an increase in the chairside time, makes isolation of the area from saliva more problematic and also increases the expenses involved ${ }^{4}$. Also, primers are likely irritants 5,6 .

These limitations of conventional composites have compelled researchers to develop newer generation primerless flowable adhesives ${ }^{7}$, which do not require an additional step for priming, thus resulting in the procedure being completed with less chairside time $^{8}$, the number of steps involved in bonding being reduced, sufficient working time, handling of the adhesive being made easier 9 , easier to control and maintain isolation against saliva and also these adhesives provide fluoride release for protection against caries ${ }^{10}$. Furthermore, the primer part; which has possible allergenic properties, is eliminated in these adhesives.

An in vitro study 7 aiming to assess the shear bond strength values of these materials concluded that primerless flowable adhesivescould be used for daily clinical use because of sufficient bond strength. Bazargani et al, while comparing lingual retainers bonded with or without primer, found failure rate to be on the higher level in the no primer group $(27 \%)$ when compared to the primer containing group $(4 \%)$ which was deemed statistically significant by the authors who recommended bondingretainers with primer ${ }^{2}$. 
Evidence on this matter is not substantial since most of the studies are in vitro and are based on bonding of brackets, while fewer studies have been conducted regarding bonding of lingual retainers and therefore further studies are needed in this regard.

This study therefore was designed to compare the lingual retainers bonded with primerless flowable adhesives and conventional bonding adhesive, in terms of early clinical failure.

\section{METHODOLOGY}

The study was approved by ethics review committee (ref. no. 905/Trg-ABP1K2) of Armed Forces Institute of Dentistry (AFID). It was a quasi-experimental study where seventy-six patients who had completed orthodontic treatment in AFID were selected on a nonprobability consecutive method. Sample size was calculated using WHO sample size calculator. By keeping the levelofsignificance as $5 \%$, power of test as $80 \%$, anticipated population of failure in conventional bonding adhesive group as 0.04 and anticipated population of failure in primerless flowableadhesive group as 0.27 , a sample size of 38 was calculated for each group making a total of $76^{2}$.

Patients between the ages of 13-25 years of age requiring fixed retention, with all anterior dentition present and sound enamel on the lingual surface were included in this study. Exclusion criteria was, patients having malaligned lower incisors with significant marginal ridge discrepancy, rotated canines, spacing, presence of acute gingivitis, and presence of caries or enamel defects.

Informed consent was obtained from the patients. Patients were randomly divided into two groups: the conventional bonding adhesive $(n=38)$ and the primerless flowable adhesive group $(n=38)$. Scaling and polishing of the lingual surface of lower anterior teeth was done 24 hours prior to the application of lingual retainer. The same clinician bonded all the retainers. The retainer wire was gently adapted against the teeth to determine the length required. Isolation was achieved with help of cotton rolls and rapid suction to prevent salivary contamination. Thirty seven percent phosphoric acid (Meta Etchant) was applied for 30 seconds on lingual surface of lower incisors followed by careful rinsing and then air dried. The wire was then adapted against the lingual surfaces and stabilized in position with the help of dental floss.

In the conventional bonding adhesive group, acoating of primer (Prime \& Bond NT; Dentsply) wasbrushed to the inner teeth surfaces and then light cured using LED curing light (3M ESPE Elipar) for 30 seconds. Small amount of composite (Spectrum Dentsply) was then placed on the already primercoated surfaces ensuring maximum coverage of the wire surface followed by light curing using LED curing light with a duration of 30 seconds per tooth.

In the primerless flowable adhesive group, the adhesive (Heliosit Orthodontic; Ivoclar) was directly applied to the lingual surface without any intermediate primer step, ensuring maximum coverage of the wire surface followed by light curing using LED curing light with a duration of 30 seconds per tooth.

Following retainer placement, the patients were given postoperative oral hygiene instructions. The bonded retainer wasthen evaluated 3 months after placement. Any bonding failures were recorded.

The statistical analysis was carried out using statistical software (version 23; SPSS). Frequency and percentage were calculated for qualitative variables for example gender, failure of retainer. Mean and standard deviation were calculated for quantitative variables like age. Fisher's exact test was used to compare frequency of failures between the two bonding adhesives. $p$-value of $\leq 0.05$ was considered as significant.

\section{RESULTS}

The study included 76 patients $(n=76)$ among which $40(52.6 \%)$ were female and $36(47.4 \%)$ were male patients. The age of the patients ranged from 13-25 years with an average age of $16.7 \pm 3.1$ years. Fig-1, shows the scatter of ages along with frequency within the sample.

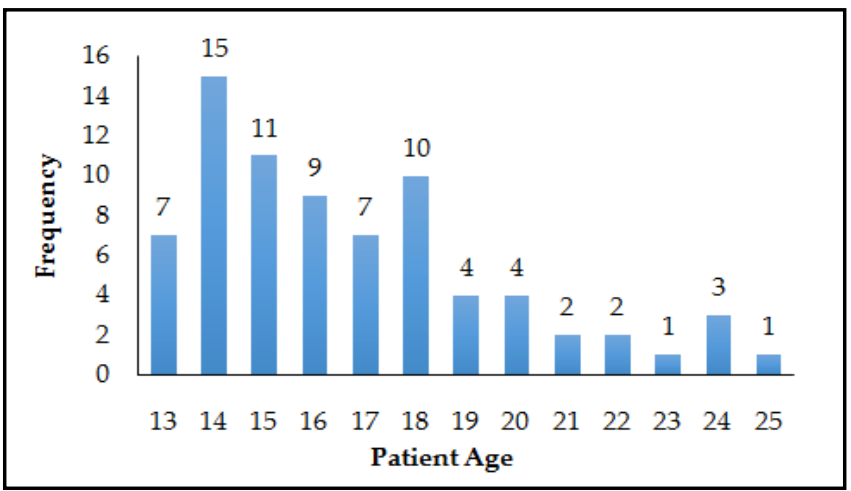

Figure-1: Scatter of ages with frequency within sample.

In the primer-less flowable adhesive group, failure was observed in 12 patients (31.6\%). In the conventional bonding adhesive group, the failure was observed in 4 patients (10.5\%). Fig-2, showed the frequency 


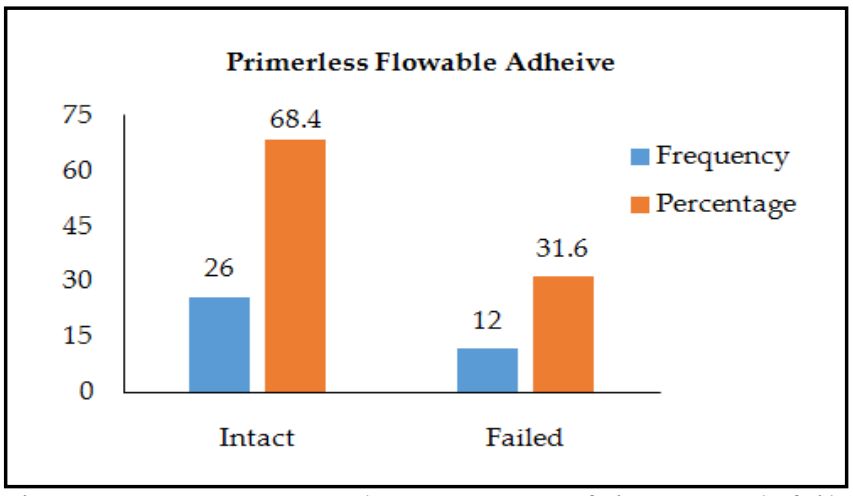

Figure-2: Frequency and percentage of intact and failed retainersin the primer-less flowable adhesive group.

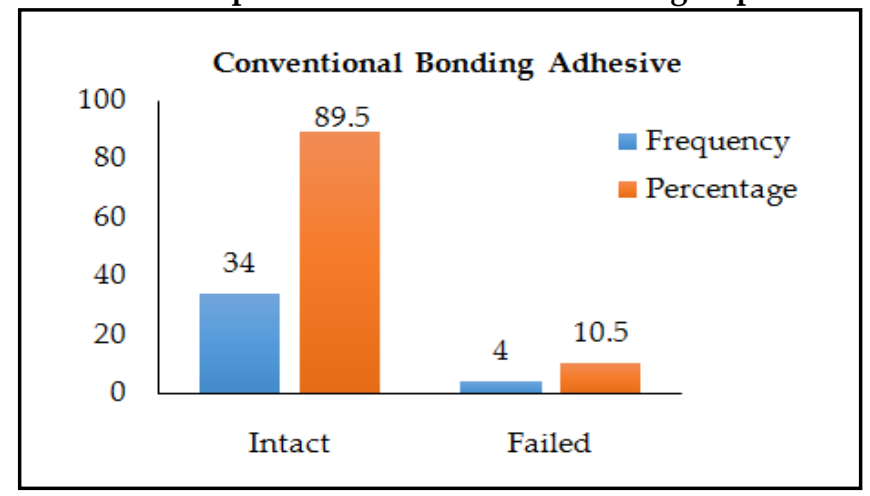

Figure-3: Frequency and percentage of intact and failed retainersin the conventional bonding adhesive group.

and percentage of intact and failed retainers in the primerless flowable adhesive group. Fig-3, displayed the frequency and failure of intact and failedretainers in the conventional bonding adhesive group.

The failure rates between the two groups was compared using Fisher's exact test, and a $p$-value of $<0.05$ was considered statistically significant. The $p$ value turned out to be 0.047 , thus the null hypothesis is rejected. Results are summarized in table.

Table: Comparison of failure rates of retainers between both groups.

\begin{tabular}{l|c|c}
\hline Study Group & $\begin{array}{c}\text { Number of } \\
\text { Failures (\%) }\end{array}$ & $p$-value \\
\hline $\begin{array}{l}\text { Primerless Flowable } \\
\text { Adhesive (n=38) }\end{array}$ & $12(31.6 \%)$ & \multirow{2}{*}{0.047} \\
\cline { 1 - 2 } $\begin{array}{l}\text { Conventional Bonding } \\
\text { Adhesive }(\mathrm{n}=38)\end{array}$ & $4(10.5 \%)$ & \\
\hline
\end{tabular}

\section{DISCUSSION}

This study was aimed at comparing lingual retainers bonded with primer less flowable adhesives versus retainers bonded with conventional bonding adhesives, in term of early clinical failure. If proven that primerless flowable adhesives have failure rates similar to that of conventional bonding adhesives, the use of primer less adhesives can be recommended because of their many favorable clinical characteristics which include, not requiring an additional step for priming and therefore resulting in less chairside time, reduction of the number of steps in bonding, sufficient working time, ease of handling, easier to maintain isolation against saliva11, and also fluoride release for protection against caries. Furthermore, the primer part; which is a potential allergen ${ }^{12,13}$, is eliminated in the primer less flowable adhesives.

The results of this study showed a high incidence of failure of $31.6 \%$ with primer less flowable adhesives and a relatively low incidence of failure of $10.5 \%$ with use of conventional bonding adhesives. This proved that there was a significant difference between the clinical failure rates with significantly greater clinical failure of lingual retainers when bonded with primer less flowable adhesives. Hence, the use of primer less flowable adhesives is not recommended for bonding lingual retainers due to the higher incidence of failure rate.

The results of this study coincide with those of Bazargani et $a l^{2}$, their results demonstrated a greater failure rate of $27 \%$ in the no primer group versus a $4 \%$ clinical failure rate in the primer containing adhesive group which was statistically significant. This allowed the authors to conclude that the lingual retainers should be bonded with adhesives containing the primer step rather than using primer-less adhesives.

The findings of this study did not coincide with that of Tang et all4. They in aretrospective study compared a chemically cured adhesives with and without theuse of primers with regards tobracket failure rate. The results were similar with bracket failure rates of $5.62 \%$ without primer and $6.22 \%$ with primer leading the authors to conclude that fixed appliancescan be bonded equally well with or without primers. However, the results were notapplicable because firstly, the study by Tang et al, usedchemically cured adhesives, where as this trial uses light cured adhesives in both groups. Secondly, the retrospective nature of study makes it less reliable as compared to prospective randomization of this trial.

Tang et $a l^{15}$, in another study concluded that lingual retainers can be effectively bonded without the use of bonding resin and therefore contradicting the results of this study. One reason for the difference between the results maybe that the authors compared the 
two groups using the same adhesive brand (Transbond LR) with the difference being that in one group the primer step was included, while in the other group the same adhesive was used except that the primer step of bonding was excluded despite the manufacturer's recommendation of using the primer which is included as part of the product. On the other hand, this trial used a conventional adhesive with primer in one group and the other group consisted of an adhesive which is specially designed as a primer-less adhesive.

Rai $e t \mathrm{al}^{4}$, in an in vivo study compared brackets bonded with or without primer resin, demonstrating a similar failure rate between both groups, which does not coincide with the results of this study. However, this study was limited by the fact that the sample size was very limited. Furthermore, this study considered failure rate of brackets rather than that of lingual retainers whereas this study was focused at bonding of lingual retainers.

In vitro studies, comparing shear bond strengths of these adhesives have been carried out. Uysal et al16, compared the shear bond strengths of three primerless flowable adhesive groups with the conventional bonding adhesives group. The results showed shear bonds strength for the primer-less flowable adhesive group which was considered as just adequate, whereas the conventional bonding adhesive group showedgood to excellent shear bond strengths values. These results seem to correspond to the results of this study since it was probable that good shear bond strength would be highly correlated to a reduction in the clinical failure rates of conventional bonding adhesives.

The retainers were evaluated for clinical failure after a follow-up of 3 months. However, clinically for retention purposes, retainer wires are usually kept in place for a minimum of 2 years after completion of orthodontic treatment to maintain proper alignment of teeth and prevent relapse ${ }^{17}$. Therefore, further studies on this subject can be undertaken with longer followup periods which would allow a more thorough comparison between the clinical failure rates of these two groups of materials.

Additionally, in terms of retainer placement, adapting and bending the retainer wire on the study cast instead of directly bending the wire intra orally would helpachieve good adaptation of retainer wire on the lingual surface of anterior teeth, and would ensure thatthe retainer wire is passive, thus ensuring that noinadvertent tooth movement18is caused, and undue stresses are applied on theretainer-wire interface.
Lastly, future studies on this subject may use other brands of primer less flowable adhesives and conventional bonding adhesives. It may be possible that adhesives from other brands may show difference in results due to their unique formulations with relation to filler content,degree of polymerization and other parameters.

\section{CONCLUSION}

A statistically significant difference was found between the clinical failure rates of lingual retainers bonded with primer less flowable adhesives versus conventional bonding adhesives with a significantly increased clinical failure rate of lingual retainers which are bonded with primer less flowable adhesives. Therefore, based on the results of this study, it is recommended to use conventional bonding adhesives for the bonding of lingual retainers due to their reduced clinical failure rate, despite the many advantages of avoiding the primer step when bonding with primer less flowable adhesives.

\section{CONFLICT OF INTEREST}

This study has no conflict of interest to be declared by any author.

\section{REFERENCES}

1. Littlewood SJ. Evidence-based retention: Where are we now? Semin Orthod 2017; 23(2): 229-36.

2. Bazargani F, Jacobson S, Lennartsson B. A comparative evaluation of lingual retainer failure bonded with or without liquid resin. Angle Orthod 2012; 82(1): 84-87.

3. Bazargani F, Magnuson A, Löthgren H, Kowalczyk A. Orthodontic bonding with and without primer: a randomized controlled trial. Eur J Orthod 2016; 38(5): 503-07.

4. Rai AK. Evaluation of bracket failure rate in orthodontic patients bonded with and without primer. Saudi J Dent Res 2015; 6(1): 48-53.

5. Tang AT, Li J, Ekstrand J, Liu Y. Cytotoxicity tests of in situ polymerized resins: methodologicalcomparisons and introduction ofa tissue culture insert as a testing device. J Biomed Mater Res 1999; 45(3): 214-22.

6. Ruyter IE, Oysaed H. Conversion in different depths of ultravioletand visible lightactivated composite materials. Acta Odontol Scand 1982; 40(3): 179-92.

7. Durrani OK, Arshad N, Rasool G, Bashir U, Kundi IU, Shaheed S. In vitro comparision of shear bond strength of transbondxt and heliosit orthodontic as direct bracket bonding adhesive. Pak Oral Den J 2008; 28(2): 203-06.

8. Thekiya AH, Aileni KR, Rachala MR, Reddy SD, Devi KS. An Evaluation of Shear Bond Strength of Admira (Ormocer) as an Alternative Material for Bonding Orthodontic Brackets: An In vitro Study. J Int Soc Prev Community Dent 2018; 8(1): 56-61.

9. Krishnan S, Pandian S, Rajagopal R. Six-month bracket failure rate with a flowable composite: A split-mouth randomized controlled trial. Dental Press J Orthod 2017; 22(2): 69-76.

10. Chitra P, Yashpal.A Comparison of the Efficacy of a Primerless Orthodontic Bonding Adhesive as Compared to Conventional Materials: an Invitro Study. DJAS 2016: 4(1): 49-55. 
11. Mohan A, Ali H, Deepak. Orthodontic Relapse. Paripex-Ind J Res 2018; 6(12): 541-42.

12. Altmann ASP, Degrazia FW, Celeste RK, Leitune VCB, Samuel SMW, Collares FM. Orthodontic bracket bonding without previous adhesivepriming: A meta-regression analysis. Angle Orthod 2016; 86(3): 391-98.

13. Tosic G. Occupational hazards in dentistry-part one: allergic reactions todental restorative materials and latex sensitivity. Work Living Env Prot 2004; 2(4): 317-24.

14. Tang AT, Björkman L, Lindbäck KF, Andlin-Sobocki A, Ekstrand J. Retrospective study of orthodontic bonding without liquid resin. Am J Orthod Dent Orthop Off Publ Am Assoc Orthod Its Const Soc Am Board Orthod 2000; 118(3): 300-06.
15. Tang ATH, Forsberg CM, Andlin-Sobocki A, Ekstrand J, Hägg U. Lingualre tainers bonded without liquid resin: A 5-year followup study. Am J Orthod Dent Ortho 2013; 143(1): 101-04.

16. Uysal T, Sari Z, Demir A. Are the Flowable Composites Suitable for Orthodontic Bracket Bonding?. Angle Orthod 2004; 74(5): 697-02.

17. Renkema AM, Al-Assad S, Bronkhorst E, Weindel S, Katsaros C, Lisson JA. Effectiveness of lingual retainers bonded to the canines in preventing mandibular incisorrelapse. Am J Orthod Dentofacial Orthop 2008; 134(2): 179-86.

18. Shaughnessy TG, Proffit WR, Samara SA. Inadvertent tooth movement with fixed lingual retainers. Am J Orthod Dent Orthopaed 2016; 149(2): 277-86 